\title{
IL-8-251T>A (rs4073) Polymorphism Is Associated with Prognosis in Gastric Cancer Patients
}

\author{
PATRICIO GONZALEZ-HORMAZABAL ${ }^{1}$, SANDRA ROMERO ${ }^{1}$, MAHER MUSLEH ${ }^{2,3}$, \\ MARCO BUSTAMANTE ${ }^{4}$, JUAN STAMBUK ${ }^{5}$, RAUL PISANO ${ }^{6}$, ENRIQUE LANZARINI ${ }^{2,3}$, \\ HECTOR CHIONG ${ }^{7}$, JORGE ROJAS $^{3}$, VICTOR GONZALO CASTRO ${ }^{1}$, LILIAN JARA ${ }^{1}$ and ZOLTAN BERGER ${ }^{2}$ \\ ${ }^{1}$ Human Genetics Program, Institute of Biomedical Sciences (ICBM), \\ School of Medicine, University of Chile, Santiago, Chile; \\ ${ }^{2}$ Department of Gastroenterology, University of Chile Clinical Hospital, Santiago, Chile; \\ ${ }^{3}$ Department of Surgery, University of Chile Clinical Hospital, Santiago, Chile; \\ ${ }^{4}$ Department of Surgery, School of Medicine at Eastern Campus, University of Chile, Santiago, Chile; \\ ${ }^{5}$ Department of Surgery, San Juan de Dios Hospital, Santiago, Chile; \\ ${ }^{6}$ Department of Pathology, San Juan de Dios Hospital, Santiago, Chile; \\ ${ }^{7}$ Department of Surgery, Barros Luco Hospital, Santiago, Chile
}

\begin{abstract}
Background/Aim: Inflammation is a key process in gastric carcinogenesis. Cytokines are mediators of inflammation and are involved in metastasis and tumorigenicity. We previously assessed the role of cytokine gene polymorphisms in gastric cancer risk in Chile. In the present study, we aimed to analyze whether these polymorphisms are associated with overall survival $(O S)$ in gastric cancer $(G C)$ patients. Patients and Methods: A total of 153 individuals with GC diagnosis were followed-up for at least 2 years. Hazard ratios (HR) were estimated from Cox regression models using SNPs as predictor variables. The following SNPs were genotyped for study using a TaqMan assay: $r$ s16944 (IL1B -511C $>$ T); rs4073 (IL8 -251 $T>A$ ); rs2275913 (IL-17 -197G>A); rs1800872 (IL10 -592 $C>A$ ); $r s 1800896$ (IL10 -1082A>G); rs28372698 (IL32). Results: Interleukin-8 rs4073 (IL-8 $-251 T>A)$ showed association with $O S$ under the dominant model $(T A+A A)$ only when adjusted by clinicopathological variables (HR=1.64, $95 \% C I=1.05-2.55, p=0.030, q$-value $=0.18)$, but not with the univariate model $(H R=1.51,95 \% C I=0.98-2.31, p=0.062, q$ value $=0.37)$. No significant differences were observed after adjusting for population stratification (PC1 and PC2 from
\end{abstract}

This article is freely accessible online.

Correspondence to: Patricio Gonzalez-Hormazabal, Human Genetics Program, Institute of Biomedical Sciences (ICBM), School of Medicine, University of Chile, Av. Independencia 1027, Santiago, Chile. Tel: +56 229786845, e-mail: patriciogonzalez@uchile.cl

Key Words: Gastric cancer, polymorphisms, SNP, interleukin-8, prognosis, survival.
Principal Component Analysis using genotypes from Infinium Global Screening Array). After stratification by clinicopathological variables, the association with shorter overall survival was higher among patients with diffuse-type tumors $(H R=2.24,95 \% C I=1.16-4.45)$ and patients with tumor size $>5 \mathrm{~cm}(H R=1.79,95 \% \mathrm{CI}=1.08-2.97)$. Conclusion: These results suggest a role of $I L-8$ rs4073 in cancer prognosis. Its use as a prognostic marker of GC survival warrants further investigation.

Gastric cancer is the third leading cause of cancer-related deaths worldwide, after lung and liver cancers (1). Overall survival (OS) at 5 years decreases dramatically as the TNM stage progresses, dropping from $94.5 \%$ for stage IA to $18.7 \%$ for stage IIIC (2). This is particularly important in Latin America, where the high mortality rate is linked to advanced-stage diagnoses. For instance, in Chile it was estimated that $20.8 \%$ of patients were diagnosed at stage III and $37.6 \%$ of patients at stage IV (3). There is growing evidence associating genetic polymorphisms with gastric cancer (GC) survival, which makes SNPs attractive biomarkers to help predict prognosis.

Inflammation is a biological response to tissue injury or infection. It is manifested by the mobilization of immune cells, induction of angiogenesis, and alterations in the connective tissue, all contributing to tissue repair or removal of the pathogen (4). The "Correa's cascade" describes the progression from gastritis to atrophic gastritis, metaplasia, dysplasia and finally adenocarcinoma, with inflammation occurring very early in gastric carcinogenesis (4). The inflammatory response is mediated by cytokines, including interleukins, chemokines and lymphokines, some of which have demonstrated involvement in metastasis and tumorigenicity (4). 
El-Omar et al. (5) were the first to propose that functional polymorphisms in the interleukin-1 beta gene $(I L 1 B)$ and interleukin-1 receptor antagonist gene $(I L I R N)$ are associated with increased risk of GC. Thereafter, various studies have assessed the association of GC with polymorphisms in genes encoding cytokines $(6,7)$. Mocelin et al. (8) performed a systematic analysis of all studies analyzing SNPs associated with GC, and found 18 SNPs belonging to genes involved in immunity and inflammation, representing $36 \%$ of all associated SNPs. Nevertheless, there are few published studies that address if these SNPs are also associated with prognosis in GC patients (9-12).

We previously published the results of a case-control study assessing the role of cytokine gene polymorphisms in gastric cancer risk in Chile (13). In the present study, we aimed to analyze whether those polymorphisms are associated with $\mathrm{OS}$ in GC patients.

\section{Patients and Methods}

Patients. A total of 153 individuals with a preoperative diagnosis of gastric adenocarcinoma were recruited, clinically characterized, and later followed-up as fully described by Romero et al. (14).

Ethical approval. This study was approved by the Ethical Committee of the following institutions: University of Chile School of Medicine (\#023/2011), University of Chile Clinical Hospital (\#029/2011), Metropolitan South-Santiago Public Health Agency (\#MK523B-118), Metropolitan East-Santiago Public Health Agency (\#24/01/2012), and Metropolitan West-Santiago Public Health Agency (\#236/2009). All participants gave their written informed consent. The study was performed in accordance with the Declaration of Helsinki.

Genotyping. Genotyping of single nucleotide polymorphisms (SNPs) was carried out by TaqMan single nucleotide polymorphism assay as previously described (13). The following SNPs were studied: rs16944 (IL1B $-511 \mathrm{C}>\mathrm{T})$; $r$ rs4073 (IL8 $-251 \quad \mathrm{~T}>\mathrm{A})$; rs2275913 (IL-17 $-197 \mathrm{G}>\mathrm{A})$; rs1800872 (IL10 -592 C $>\mathrm{A})$; rs1800896 (IL10 -1082A $>$ G); rs28372698 (IL32). Given the calculations obtained using sim.snp.expsurv.power function included in survSNP package ( $\mathrm{R}$ statistical environment), a power $>0.8$ assuming an effect of Hazard Ratio (HR) $=1.7$ could be reached by analyzing SNPs with a minor allele frequency (MAF) $>0.20$. Therefore, rs1143634 (IL1B 3954C >T, MAF=0.14), rs763780 (IL17F p.His161Arg, MAF=0.03) and rs1800629 (TNF -308G >A, $\mathrm{MAF}=0.08)$ were not included in the study because the MAF was $<0.20$. rs1143627 (ILIB -31 T>C) was not considered because is in strong linkage disequilibrium with rs 16944.

Statistical analyses. HR was estimated from Cox regression models using SNPs as predictors. The assumption of proportional hazards was tested according to Gramsch and Therneau (15) for all the selected variables. For SNPs, allele (additive), dominant and recessive models were considered. To infer population stratification, we used the set of genotypes obtained from Infinium Global Screening Array (Illumina, CA, USA) as mentioned in (14). The guideline of Anderson et al. (16) was used to filter data to perform

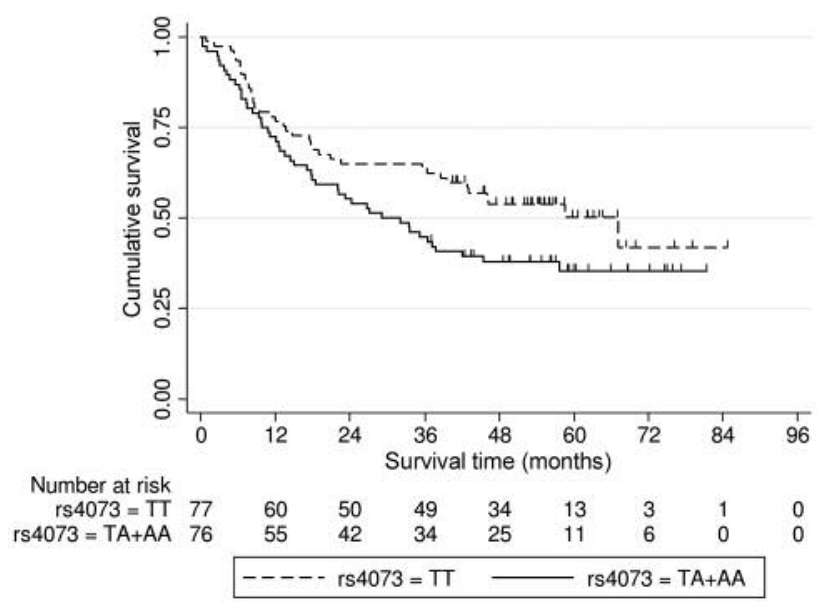

Figure 1. Kaplan-Meier curves of overall survival for rs4073 (IL-8) in gastric cancer patients.

a principal component analysis (PCA) using the routine --pca implemented in plink 1.9 (REF). Principal component 1 (PC1) and PC2 was used to adjust for population stratification in Cox regression models. False discovery rate (FDR) was used to correct for multiple testing and q-value was calculated using a spreadsheet (http://www.biostathandbook.com/bonferroni.xls) according to the Benjamini-Hochberg procedure (17). The association of polymorphisms with survival was also assessed with the log-rank test and Kaplan-Meier method. Median Survival Time (MST) was estimated at the 50th percentile, or otherwise indicated. The Hausman specification test (18) was used to assess differences in beta coefficients from two Cox regression models. Power (beta error) for estimation of HR was calculated with sim.snp.expsurv.power function included in 'survSNP' package version 0.24 using $\mathrm{R}$ statistical environment ( $\mathrm{R}$ version 3.5.1). $p$-Values were 2 -sided and $p<0.05$ was considered statistically significant. All statistical analyses were performed using Stata 12 (StataCorp LLC, TX, USA).

\section{Results}

Demographic and clinicopathological characteristics of the studied patients and their association with OS are fully described in (14). All polymorphisms were assessed for association with OS under the additive, dominant and recessive models. The only polymorphism significantly associated with OS was IL-8 rs4073. This polymorphism showed association with OS under the dominant model (TA + AA) only when adjusted by clinicopathological variables $(\mathrm{HR}=1.64,95 \% \mathrm{CI}=1.05-2.55, \quad p=0.030, \quad \mathrm{q}$-value $=0.18$, power=0.98), but not in the univariate model $(\mathrm{HR}=1.51$, $95 \% \mathrm{CI}=0.98-2.31, p=0.062$, q-value $=0.37)$. Comparisons of Kaplan-Meier estimator curves for rs4073 TA + AA versus TT genotypes are shown in Figure 1. When population stratification was included as a confounding variable (PC1 and PC2), the strength of association adjusted by clinicopathological variables was $\mathrm{HR}=1.60$ (95\% CI $=1.04$ - 
Table I. Hazard Risks of rs4073 (IL-8) according to patient's characteristics.

\begin{tabular}{|c|c|c|c|c|}
\hline Strata & $\operatorname{HR}(95 \% \mathrm{CI})^{\mathrm{a}}$ & $p$-Value & Powere & Hausman $p$-Value \\
\hline All patients $(n=153)$ & $1.49(0.91-2.30)$ & 0.067 & - & \\
\hline Intestinal-type $(n=100)$ & $1.20(0.68-2.11)$ & 0.524 & - & $0.0007^{b} ; 0.007^{c}$ \\
\hline Diffuse-type $(n=53)$ & $2.24(1.16-4.45)$ & 0.017 & 0.96 & \\
\hline Tumor size $<5 \mathrm{~cm}(\mathrm{n}=53)$ & $0.93(0.39-2.21)$ & 0.869 & - & $0.045^{\mathrm{b} ;} 0.030^{\mathrm{d}}$ \\
\hline Tumor size $>5 \mathrm{~cm}(\mathrm{n}=100)$ & $1.79(1.08-2.97)$ & 0.023 & 0.96 & \\
\hline
\end{tabular}

${ }^{\mathrm{a}} \mathrm{HR}(95 \% \mathrm{CI})$ : Hazard Risk (95\% Confidence Interval) adjusted for age and sex; ${ }^{\mathrm{b}} \mathrm{Hausman} p$-Value comparing both strata; ${ }^{\mathrm{c}} \mathrm{Hausman} p$-Value

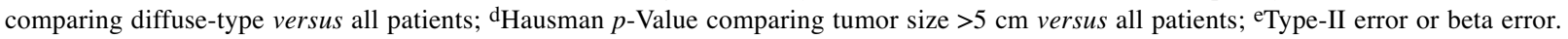

2.50, $p=0.034, \mathrm{q}=0.20$ ), and under the univariate model was $\mathrm{HR}=1.49 \quad(95 \% \mathrm{CI}=0.97-2.30, p=0.070, \mathrm{q}=0.49)$. These results suggest that this polymorphism could modify OS depending on the clinicopathological features of the patient.

The prognostic value of rs 4073 was assessed by stratified analysis of clinicopathological features. Differences in HR were observed stratifying by Lauren's classification and by tumor size. As noted in Table I, a significantly higher HR was observed among patients with diffuse tumors $(n=53)$ as compared to intestinal-type tumors $(\mathrm{n}=100)$ (Hausman $p$ value $=0.0007)$. The OS was also less when patients had a tumor $>5 \mathrm{~cm}(\mathrm{n}=100)$ compared to those with tumors $<5 \mathrm{~cm}$ $(\mathrm{n}=53)$ (Hausman $p$-value=0.045). Taken together, allele A of rs4073 conferred a low OS among gastric cancer patients, in particular those with diffuse-type tumors and size $>5 \mathrm{~cm}$.

\section{Discussion}

Few studies have addressed the contribution of rs4073 on cancer prognosis. Snoussi et al. found a significant association between the IL-8 -251 A allele with decreased OS and diseasefree survival in breast carcinoma patients from Tunisia (19, 20). Lurje et al. (11) studied whether 11 polymorphisms of genes involved in angiogenesis were related to survival in 137 GC patients from the United States. They found two polymorphisms associated with OS: IL-8 rs4073 $(p<0.0001)$ and PAR-1 rs11267092 ( $p=0.005)$, with only the former showing association when adjusted for clinicopathological variables $(\mathrm{HR}=2.45,95 \% \mathrm{CI}=1.02-5.88, p=0.045)$.

The polymorphism rs4073 is a T $>$ A substitution, 352 base pairs upstream from the start triplet (c.-352T>A) and 153 base pairs upstream from the transcription start site (TSS) of IL-8 gene. Since rs4073 has been associated not only with cancer but also other diseases, various authors have assessed the phenotypical consequences of this nucleotide substitution at the cellular and molecular level. Elevated levels of mRNA were associated with rs4073-A allele in stimulated lymphoblastoid cell lines (21). Andia et al. also found a heightened expression of IL-8 among subjects with AT genotype compared to those with TT genotype (22) in gingiva from chronic periodontitis patients. In blood samples from healthy donors, IL-8 levels were measured by ELISA in whole blood stimulated with LPS (23), where IL-8 production was significantly higher in rs4073-A carriers than in TT homozygous. Some investigations regarding whether rs4073 substitution affects promoter activity by luciferase gene reporter assay have reported contradictory results. Ahn et al. (24), using 293T cells and Lee et al. (25), using MKN45 and SC-M1 gastric cancer cell lines found that the promoter activity of $-251 \mathrm{~T}$ allele was stronger than $-251 \mathrm{~A}$ allele. On the contrary, Ohyauchi et al. (26) found with AGS cells that $-251 \mathrm{~A}$ promoter showed significantly higher luciferase activity than the $-251 \mathrm{~T}$ promoter. In the first study, a reporter plasmid was constructed using PCR fragments from Chinese patients, but in the latter Ohyauchi et al., used PCR fragments from Japanese patients. This difference could account for differences in the haplotype context of rs 4073 among Japanese and Chinese subjects (27).

There exist certain tools for annotation of functional variations intended to predict the effect of common noncoding variations. According to the Genotype-Tissue Expression (GTEx) project (www.gtexportal.org), rs4073 is not an expression quantitative trait locus (eQTL) for IL-8. Nevertheless, it is for CXCL6, which is also located on chromosome 4. In this case, rs4073-A allele is associated with increased expression of CXCL6. In fact, the gene also encodes a chemokine secreted by endothelial cells resulting in tumor development, invasion and metastasis (28). The possibility that rs4073 could be associated with cancer by increasing expression of CXCL6 deserves further investigation. Analyses of HaploReg v4.1 using data from Roadmap Epigenomic Consortium indicate that this SNP lies in an enhancer region (state 2 and 7 of Core 15 ChromHMM states) and in DNAse hypersensitive sites. Non-coding associated variants from GWAS studies are enriched in enhancer and DNAse hypersensitive sites $(29,30)$, therefore, a SNP present in these sites is likely functional. According to data from the ENCyclopedia of DNA Elements ENCODE- retrieved from RegulomeDB (31), rs4073 is located in binding sites for transcription factors TFAP2A, 
TFAP2C, JUND and JUN in HeLa-S3 cells. Taken together, the bioinformatic evidence above supports a functional effect of rs 4073 .

It has been demonstrated that various carcinomas including those of the breast, colon, cervix, stomach, lung and ovary express high levels of IL-8 relative to normal tissue (32). According to data from The Cancer Genome Atlas (TCGA) project (retrieved from Gene Expression Profiling Interactive Analysis -GEPIA- (33)), IL-8 is significantly overexpressed in gastric cancer tumors as compared to normal gastric mucosa. However, CXCL8 (also known as IL-8) mRNA expression in 440 gastric tumors from TCGA PanCancer Atlas (obtained at cBioPortal www.cbioportal.org) does not show association with Lauren's classification or TNM stage. A direct correlation between IL-8 serum levels and progression has been described for various cancers (32). According to TCGA data analysis performed in OncoLnc (34), CXCL8 mRNA levels are associated with low OS in three tumors: Cervical squamous cell carcinoma and endocervical adenocarcinoma $(\mathrm{HR}=1.64, p$ value $=0.00059)$, kidney renal clear cell carcinoma $(H R=1.26$, $p$-value $=0.0031)$, and liver hepatocellular carcinoma $(\mathrm{HR}=1.30$, $p$-value $=0.0032)$. Using the gastric cancer set from TCGA PanCancer Atlas, we retrieved OS and expression level (classified as low expression $\leq 75 \%$, otherwise high expression) data for samples with a minimum of one year of follow-up ( $n=205 / 440$ total subjects). The association of IL-8 tumor levels with OS was not significant $(\mathrm{HR}=1.27$, 95\% CI $=0.90-1.80)$ even after adjusting for clinicopathological variables $(\mathrm{HR}=1.11,95 \% \mathrm{CI}=0.72-1.73)$

Interleukin-8 (CXCL8) is a chemokine that belongs to the CXC subfamily (35). It is considered pro-inflammatory due to its role as a chemoattractant for neutrophils, but increasing evidence has linked IL-8 with cancer. More than two decades ago, Koch et al. experimentally demonstrated that IL-8 is an angiogenic factor and that also induces proliferation and chemotaxis of human umbilical vein endothelial cells (36). In 1998, Kitadai et al. was the first to link IL-8 with gastric cancer metastasis. They first found that expression of IL-8 in gastric cancer tumors is directly correlated with blood vessel count in gastric carcinomas (37). After, they injected gastric cancer cells ectopically expressing IL-8 into the stomachs of nude mice, and observed that cells expressing the chemokine grew and induced angiogenesis more than injected gastric cancer cells not ectopically expressing IL-8 (38). It has been proposed that the effect of IL-8 on angiogenesis is mediated by the expression of VEGF-A and VEGFR-2 (39). Kuai et al. (40) experimentally studied the effect of IL-8 on promoting adhesion, migration and invasion in gastric cancer cell lines. MKN-45 cells ectopically expressing IL-8 showed increased levels of those three phenotypes. In KATO-III cells, which endogenously overexpress IL-8, the treatment with siRNA against $I L-8$ gene decreased rates of adhesion, migration and invasion.
Epithelial-to-mesenchymal transition (EMT) is associated with negative prognosis in GC patients. Murai et al. (41) found that patients with gastric tumors classified as "mesenchymal" (high ratio of vimentin/E-cadherin mRNA in tumor) had a significantly lower OS than "epithelial" tumors (low ratio of vimentin/E-cadherin mRNA in tumor). Fernando et al. (42) was the first to demonstrate that IL-8 also has a key role in EMT, using breast cancer cell lines. Through in vivo and in vitro experiments, Chung et al. (43) demonstrated that the extracellular high-mobility group box-1 (HMGB1) induces EMT in GC using N87, MKN28, SNU-1 and KATOIII GC cell lines as well as in an animal model. IL-8 is a major HMGB1-inducible soluble mediator of EMT. In fact, they observed that suppression of IL-8 reversed HMGB1induced EMT in GC cells. To the best of our knowledge, no other studies have been published addressing the role of IL-8 in EMT in GC. This area deserves further investigation.

In conclusion, our study found that IL-8 rs4073 is associated with OS in GC patients. The association was more noticeable among patients with large tumors and patients with diffuse-type tumors. Both experimental and database information allowed us to propose that rs4073 is a functional polymorphism. Evidence from Lurje et al. (11) combined with our results lead us to propose a role for rs4073 in GC prognosis. Its use as a prognostic marker for GC survival warrants further investigation.

\section{Acknowledgements}

The Authors would like to acknowledge BSc Tracy Wormwood for her help in proofreading the manuscript. This work was supported by Fondo Nacional de Desarrollo Científico y Tecnológico -Chile(FONDECYT) \#1151015.

\section{References}

1 Ferlay J, Soerjomataram I, Dikshit R, Eser S, Mathers C, Rebelo M, Parkin DM, Forman D and Bray F: Cancer incidence and mortality worldwide: Sources, methods and major patterns in GLOBOCAN 2012: Globocan 2012. Int J Cancer 136: E359E386, 2015.

2 Ji X, Bu ZD, Yan Y, Li ZY, Wu AW, Zhang LH, Zhang J, Wu XJ, Zong XL, Li SX, Shan F, Jia ZY and Ji JF: The 8th edition of the American Joint Committee on Cancer tumor-nodemetastasis staging system for gastric cancer is superior to the 7th edition: results from a Chinese mono-institutional study of 1663 patients. Gastric Cancer 21: 643-652, 2018.

3 MüLler B, De La Fuente HH, Barajas B O, Cardemil JB, Vila TA, Mordojovich SE, Peñ AN K, Castillo CC, Campos MM, Rojas RH, Quijada PI and Yáñez RC: Registro de evaluación de tratamiento de cáncer gástrico en Chile (REGATE): Características clínicas básales de 523 pacientes. Rev Chil Cir 63: 147-153, 2011.

4 Yao M, Brummer G, Acevedo D and Cheng N: Cytokine Regulation of Metastasis and Tumorigenicity. In: Advances in Cancer Research. Elsevier, pp. 265-367, 2016. 
5 El-Omar EM, Carrington M, Chow W-H, McColl KEL, Bream JH, Young HA, Herrera J, Lissowska J, Yuan C-C, Rothman N, Lanyon G, Martin M, Fraumeni JF and Rabkin CS: Interleukin1 polymorphisms associated with increased risk of gastric cancer. Nature 404: 398-402, 2000

6 Persson C, Canedo P, Machado JC, El-Omar EM and Forman D: Polymorphisms in inflammatory response genes and their association with gastric cancer: a huge systematic review and meta-analyses. Am J Epidemiol 173: 259-270, 2011.

7 Vianna JS, Almeida da Silva PE and Bastos Ramis I: Helicobacter pylori pathogenicity genes, cytokine polymorphisms and environmental factors affect the development of gastric diseases: An overview. R Epidemiol Control Infec 6: 211-219, 2016.

8 Mocellin S, Verdi D, Pooley KA and Nitti D: Genetic variation and gastric cancer risk: a field synopsis and meta-analysis. Gut 64: 1209-1219, 2015

9 Graziano F, Ruzzo A, Santini D, Humar B, Tonini G, Catalano V, Berardi R, Pizzagalli F, Arduini F, Bearzi I, Scartozzi M, Cascinu S, Testa E, Ficarelli R and Magnani M: Prognostic role of Interleukin-1 $\beta$ Gene and Interleukin-1 Receptor Antagonist gene polymorphisms in patients with advanced gastric cancer. $\mathrm{J}$ Clin Oncol 23: 2339-2345, 2005.

10 Liu J: Polymorphisms of interleukin-10 promoter are not associated with prognosis of advanced gastric cancer. World $\mathrm{J}$ Gastroenterol 17: 1362, 2011.

11 Lurje G, Husain H, Power DG, Yang D, Groshen S, Pohl A, Zhang W, Ning Y, Manegold PC, El-Khoueiry A, Iqbal S, Tang LH, Shah MA and Lenz H-J: Genetic variations in angiogenesis pathway genes associated with clinical outcome in localized gastric adenocarcinoma. Ann Oncol 21: 78-86, 2010.

12 Ruzzo A, Catalano V, Canestrari E, Giacomini E, Santini D, Tonini G, Vincenzi B, Fiorentini G, Magnani M and Graziano F: Genetic modulation of the interleukin 6 (IL-6) system in patients with advanced gastric cancer: a background for an alternative target therapy. BMC Cancer 14: 357, 2014.

13 Gonzalez-Hormazabal P, Musleh M, Bustamante M, Stambuk J, Escandar S, Valladares H, Lanzarini E, Chiong H, Rojas J, Castro VG, Rubio-Reyes C, Jara L and Berger Z: Role of cytokine gene polymorphisms in gastric cancer risk in Chile. Anticancer Res 34: 3523-3530, 2014.

14 Romero S, Musleh M, Bustamante M, Stambuk J, Pisano R, Lanzarini E, Chiong H, Rojas J, Castro VG, Jara L, Berger Z and Gonzalez-Hormazabal P: Polymorphisms in TWIST1 and ZEB1 Are Associated with Prognosis of Gastric Cancer Patients. Anticancer Res 38: 3871-3877, 2018.

15 Grambsch PM and Therneau TM: Proportional hazards tests and diagnostics based on weighted residuals. Biometrika 81: 515526, 1994.

16 Anderson CA, Pettersson FH, Clarke GM, Cardon LR, Morris $\mathrm{AP}$ and Zondervan KT: Data quality control in genetic casecontrol association studies. Nat Protoc 5: 1564-1573, 2010.

17 Benjamini Y and Hochberg Y: Controlling the False Discovery Rate: A Practical and Powerful Approach to Multiple Testing. J R Stat Soc Series B 57: 289-300, 1995.

18 Hausman JA: Specification Tests in Econometrics. Econometrica 46: 1251-1271, 1978

19 Snoussi K, Mahfoudh W, Bouaouina N, Fekih M, Khairi H, Helal AN and Chouchane L: Combined effects of IL-8 and CXCR2gene polymorphisms on breast cancer susceptibility and aggressiveness. BMC Cancer 10: 283, 2010.
20 Snoussi K, Mahfoudh W, Bouaouina N, Ahmed SB, Helal AN and Chouchane L: Genetic variation in IL-8 associated with increased risk and poor prognosis of breast carcinoma. Hum Immunol 67: 13-21, 2006.

21 Wacharasint P, Nakada T-A, Boyd JH, Russell JA and Walley KR: AA genotype of IL-8 $-251 \mathrm{~A} / \mathrm{T}$ is associated with low $\mathrm{PaO}_{2} / \mathrm{FiO}_{2}$ in critically ill patients and with increased IL-8 expression: IL-8 SNP and $\mathrm{PaO}_{2} / \mathrm{FiO}_{2}$ ratio. Respirology 17: 1253-1260, 2012.

22 Andia DC, de Oliveira NFP, Letra AM, Nociti FH, Line SRP and de Souza AP: Interleukin-8 gene promoter polymorphism (rs4073) may contribute to chronic periodontitis. J Periodontol 82: 893-899, 2011.

23 Hull J, Thomson A and Kwiatkowski D: Association of respiratory syncytial virus bronchiolitis with the interleukin 8 gene region in UK families. Thorax 55: 1023-1027, 2000.

24 Ahn MH, Park BL, Lee SH, Park SW, Park JS, Kim DJ, Jang AS, Park JS, Shin HK, Uh ST, Kim YK, Kim YW, Han SK, Jung KS, Lee KY, Jeong SH, Park JW, Choi BW, Park IW, Chung MP, Shin HD, Song JW, Kim DS, Park CS and Shim YS: A promoter SNP rs $4073 \mathrm{~T}>\mathrm{A}$ in the common allele of the interleukin 8 gene is associated with the development of idiopathic pulmonary fibrosis via the IL-8 protein enhancing mode. Respir Res 12: 73, 2011.

25 Lee W-P: The $-251 \mathrm{~T}$ Allele of the Interleukin- 8 promoter is associated with increased risk of gastric carcinoma featuring diffuse-type histopathology in Chinese population. Clin Cancer Res 11: 6431-6441, 2005.

26 Ohyauchi M: The polymorphism interleukin 8 -251 $\mathrm{A} / \mathrm{T}$ influences the susceptibility of Helicobacter pylori related gastric diseases in the Japanese population. Gut 54: 330-335, 2005.

27 Hacking D, Knight JC, Rockett K, Brown H, Frampton J, Kwiatkowski DP, Hull $\mathrm{J}$ and Udalova IA: Increased in vivo transcription of an IL-8 haplotype associated with respiratory syncytial virus disease-susceptibility. Genes Immun 5: 274-282, 2004.

28 Gijsbers K, Gouwy M, Struyf S, Wuyts A, Proost P, Opdenakker G, Penninckx F, Ectors N, Geboes K and Van Damme J: GCP2/CXCL6 synergizes with other endothelial cell-derived chemokines in neutrophil mobilization and is associated with angiogenesis in gastrointestinal tumors. Exp Cell Res 303: 331$342,2005$.

29 Ernst J, Kheradpour P, Mikkelsen TS, Shoresh N, Ward LD, Epstein CB, Zhang X, Wang L, Issner R, Coyne M, Ku M, Durham T, Kellis M and Bernstein BE: Mapping and analysis of chromatin state dynamics in nine human cell types. Nature 473 : 43-49, 2011.

30 Schaub MA, Boyle AP, Kundaje A, Batzoglou S and Snyder M: Linking disease associations with regulatory information in the human genome. Genome Res 22: 1748-1759, 2012.

31 Boyle AP, Hong EL, Hariharan M, Cheng Y, Schaub MA, Kasowski M, Karczewski KJ, Park J, Hitz BC, Weng S, Cherry JM and Snyder M: Annotation of functional variation in personal genomes using RegulomeDB. Genome Res 22: 17901797, 2012.

32 Palena C, Hamilton DH and Fernando RI: Influence of IL-8 on the epithelial-mesenchymal transition and the tumor microenvironment. Future Oncol 8: 713-722, 2012.

33 Tang Z, Li C, Kang B, Gao G, Li C and Zhang Z: GEPIA: a web server for cancer and normal gene expression profiling and interactive analyses. Nucleic Acids Res 45: W98-W102, 2017. 
34 Anaya J: OncoLnc: linking TCGA survival data to mRNAs, miRNAs, and lncRNAs. PeerJ Computer Science 2: e67, 2016.

35 Turner MD, Nedjai B, Hurst T and Pennington DJ: Cytokines and chemokines: At the crossroads of cell signalling and inflammatory disease. Biochimica et Biophysica Acta (BBA) Mol Cell Res 1843: 2563-2582, 2014.

36 Koch AE, Polverini PJ, Kunkel SL, Harlow LA, DiPietro LA, Elner VM, Elner SG and Strieter RM: Interleukin-8 as a macrophage-derived mediator of angiogenesis. Science 258: 1798-1801, 1992.

37 Kitadai Y, Haruma K, Sumii K, Yamamoto S, Ue T, Yokozaki H, Yasui W, Ohmoto Y, Kajiyama G, Fidler IJ and Tahara E: Expression of interleukin-8 correlates with vascularity in human gastric carcinomas. Am J Pathol 152: 93-100, 1998.

38 Kitadai Y, Takahashi Y, Haruma K, Naka K, Sumii K, Yokozaki H, Yasui W, Mukaida N, Ohmoto Y, Kajiyama G, Fidler IJ and Tahara E: Transfection of interleukin-8 increases angiogenesis and tumorigenesis of human gastric carcinoma cells in nude mice. Brit J Cancer 81: 647-653, 1999.

39 Shi J and Wei PK: Interleukin-8: A potent promoter of angiogenesis in gastric cancer. Oncol Lett 11: 1043-1050, 2016.
40 Kuai WX: Interleukin-8 associates with adhesion, migration, invasion and chemosensitivity of human gastric cancer cells. World J Gastroenterol 18: 979, 2012.

41 Murai T, Yamada S, Fuchs BC, Fujii T, Nakayama G, Sugimoto H, Koike M, Fujiwara M, Tanabe KK and Kodera Y: Epithelial-tomesenchymal transition predicts prognosis in clinical gastric cancer: EMT in clinical gastric cancer. J Surg Oncol 109: 684-689, 2014.

42 Fernando RI, Castillo MD, Litzinger M, Hamilton DH and Palena C: IL-8 signaling plays a critical role in the epithelialmesenchymal transition of human carcinoma cells. Cancer Res 71: 5296-5306, 2011.

43 Chung HW, Jang S, Kim H and Lim JB: Combined targeting of high-mobility group box- 1 and interleukin- 8 to control micrometastasis potential in gastric cancer: Targeting HMGB1 and IL-8 to control EMT. Int J Cancer 137: 1598-1609, 2015.

Received August 16, 2018

Revised September 5, 2018

Accepted September 6, 2018 